\title{
Estado actual de la Educación Física desde el punto de vista del profesorado. Propuestas de mejora. Current state of Physical Education from the point of view of teachers. Improvement proposals

\author{
*Dolors Cañabate Ortíz, *Montse Tesouro Cid., *Juan Puigggali Allepuz, **María Luisa Zagalaz Sánchez
} \\ *Universidad de Girona (España), **Universidad de Jaén (España)
}

Resumen. En esta investigación el objectivo es observar la situación de la Educación Física (EF) como disciplina mediante una indagación basada en la experiencia y los conocimientos de los profesionales en activo. Se aplica un cuestionario ad hoc a 36 profesionales en activo (maestros especialistas en EF) que participan en el prácticum de la mención del alumnado de $4^{\circ}$ curso del grado de maestro/a de Primaria para que manifiesten los problemas reales de enseñanza, así como la importancia y el impacto social que tiene la EF como materia educativa. Se pretende también compartir diferentes puntos de vista de los profesionales para hacer propuestas para mejorar la formación de los profesionales de EF. Como conclusiones del estudio, se observa que la mayoría optan por la motivación como factor y condición que promueve el éxito de la EF como materia curricular y creen que se debería potenciar el trabajo cooperativo. Las características más valoradas de los profesores y profesoras de EF son: saber trabajar en equipo EF y la utilización de proyectos de innovación educativa, flexibles y adaptados al contexto, lo que supone un aprendizaje significativo. Los profesionales consideran que se debe potenciar la motricidad aplicando metodologías activas, dinámicas y reflexivas y dan mucha importancia al aprendizaje continuo. Por tanto, se proponen cursos de formación y la realización de intercambios de experiencias

Palabras clave: Educación física, educación primaria, formación, innovación educativa, conocimiento didáctico

\begin{abstract}
The present study holds the objective to observe the current state of Physical Education (PE) as a discipline through research based on the experience and the knowledge of active professionals. An ad-hoc questionnaire was completed by 36 active professionals (specialist teachers in PE) who participate in the practicum of majors of PE in the 4th year of Primary Education Bachelor. The instrument intends to bring to light the real problems of teaching, as well as the importance and the social impact that PE has as an educational subject. It also aims to share different points of view of active professionals and make proposals to improve the training of PE professionals. As conclusions of the study, most consider motivation as a factor and condition to promote the success of PE as a curricular subject and believe that cooperative work should be encouraged. The most valued characteristics of PE teachers are: knowing how to work as a PE team and the use of educational innovation projects that are flexible and adapt to the context, resulting in significant learning. Professionals consider that motor skills should be enhanced by applying active, dynamic and reflective methodologies and give great importance to ongoing learning. Therefore, proposals include training courses and exchanges of experiences.
\end{abstract}

Keywords: Physical Education, Primary Education, training, educational innovation, didactic knowledge.

\section{Introducción}

En los últimos años se ha hecho un considerable y crecienteénfasis en todo el mundo para elevar los estándares educativos y mejorar las oportunidades para todos los jóvenes. Actualmente las instituciones de educación se encuentran involucradas en un cambio orientado hacia modelos educativos de calidad y de excelencia, en donde el alumno es eje central de un proceso pertinente de enseñanza-aprendizaje (Morales, Berrocal, Morquecho \& Hernández, 2013). Una forma de lograr esto es a través de la reforma y el desarrollo curricular. La reforma y la revisión de los planes de estudios escolares se han emprendido en muchos países para educar mejor a los estudiantes. Los programas de educación física están bajo fuerte presión para demostrar que sus prácticas son efectivas para preparar a los estudiantes para una vida activa saludable y exitosa (Balázs, Susan, Dancs \& József, 2016).

Actualmente destacan las modificaciones sufridas por el sistema educativo con la intención de querer dar respuesta a las nuevas exigencias de formación, así como a las necesidades surgidas por las transformaciones sociales, tanto a nivel familiar como laboral, informativo y cultural del siglo XXI (Marchesi, 2007; Romero, 2007). Estas modificaciones han provocado que las diferentes didácticas específicas se replanteen su cometido para impulsar y responder a nuevos retos que contribuyan a las finalidades educativas actuales (Collazo, 2014). Concretamente, la formación inicial de los maestros especialistas en Educación Física (EF) ha «sufrido» profundos cambios con las reformas educativas (Delicado, Trujillo, García, 2018).

En este sentido López, Pérez, Manrique y Monjas (2016) indican que los principales retos a los que sigue enfrentándose la EF en España en este principio de siglo son tres: (a) plantearse de forma reflexiva y sistemática qué queremos que aprenda nuestro alumnado en EF; (b) tener razones claras y poderosas sobre lo que puede aportar la EF a la educación integral de las personas; y (c) lograr definitivamente la profesionalidad que venimos demandando durante los últimos 30 años.

Fecha recepción: 27-01-18. Fecha de aceptación: 14-05-18 Dolors Cañabate Ortíz dolors.canyabate@udg.edu
La EF tiene como objetivo proporcionar aprendizajes y contribuir al proceso de formación y socialización del alumnado a través de las actividades corporales (Contreras, Cuevas, Blázquez, Lleixà, Garcia, Sebastini, \& Barrachina, 2012), proporcionando una amplia gama de enseñanzas que favorezcan un desarrollo global y armónico, orientado al desarrollo de persones competentes para la vida (Romero, 2007; Blázquez \& Sebastiani, 2009; Aubert, Bizkarra \& Calvo, 2014; Monzonís \& Capllonch, 2015; López Pastor, Pérez, Manrique \& Monjas, 2016), entre otros.

En diferentes estudios se ha observado que la EF contribuye a la consecución de las competencias clave (Lleixà, Capllonch, \& González, 2015; Calderón, Martínez, \& Méndez, 2013; Bisquerra, 2011; Contreras \& Cuevas, 2011; Cañabate, 2010; Cañabate \& Zagalaz, 2010, 2011; García, 2010; Hernández, Díez, Pacheco, \& Sosa 2008, López, 2010; Lleixà, 2007; Méndez-Giménez, López-Téllez, \& SierraArizmendiarrieta, 2009; Vaca, 2008; Vicente, 2011; Zamorano, 2011). Según Zagalaz, Cachón, \& Lara (2008), entre las competencias, destaca la capacidad de análisis, síntesis y gestión de la información; la de organización y planificación; las habilidades en las relaciones interpersonales; la capacidad para la comunicación; el desarrollo crítico; la creatividad y la adaptación a las nuevas situaciones, entre otras.

Actualmente la LOMCE (2013) aboga por una enseñanza por competencias, aunque no muestra cómo desarrollarlas (RD 126/2014) ni tampoco introduce como deben ser abordadas desde la EF, no haciendo eco de la solicitud reiterada de incluir la competencia motriz entre ellas (Méndez-Giménez, López-Téller, \& Sierra Arrizmendiarrieta, 2009). Existen evidencias de que más tiempo de práctica de EF de calidad, a través de una enseñanza comprensiva, ejercería un papel importante en la promoción de la salud pública y el desarrollo integral de todo el alumnado (Méndez-Alonso, Fernández-Río, Méndez-Giménez, \& Prieto (2015); González, García, Contreras, \& Sánchez Mora, 2009; Londsdale, Rosenkranz, Peralta, Bennie, Fahey, \& Lubans, 2013).

Uno de los aspectos que determinan la identidad y calidad de la EF en la escuela es el desarrollo de competencias profesionales y la capacidad de autoeficacia alcanzado por parte del profesorado de EF (Skaalvik \& Skaalvik, 2007; Dellinger, Bobbett, Olivier, \& Ellett, 2008; Contreras, et al. 2012). Determinar cuáles son las competencias que debe tener un 
docente para que se considere de calidad es una cuestión que preocupa tanto a profesionales como a Instituciones (Díaz del Cueto \& Aguado, 2012; Baena-Extremera \& Granero-Gallegos, 2012). Hasta ahora se han realizado diferentes estudios con el objetivo de conocer la percepción sobre las competencias docentes que tiene el profesorado (Blasco, Romero, Mengual, Fernández-Revelles, Delgado, \& Vega, 2011; Del Valle, De la Vega, \& Rodríguez 2015; Hernández \& Velázquez 2010; Díaz 2009).

Un factor a tener en cuenta es la implicación de los alumnos con la escuela y la educación. Como indica González (2010)

el concepto de implicación no cuenta con una acotación conceptual unívoca, siendo entendido y manejado de diferentes modos por sus estudiosos. Diversos autores hablan de implicación «escolar», mientras otros se referirán a implicación «académica» o a implicación «en el aprendizaje», así como, también a «no-implicación» (disengagement), o desafección. Términos todos ellos que no siempre son definidos con precisión, ni si siempre son claramente discernibles entre sí. (p.13)

En estudios como el de Gutierrez (2014), se observa que los factores más relacionados con la motivación intrínseca de los alumnos han sido la percepción del clima de maestría y las experiencias en EF. Por el contrario, la percepción del clima de ejecución se ha mostrado más relacionada con la tensión-presión de los alumnos. También Cerverlló, et.al (2004) indican que la importancia del clima motivacional como variable influyente sobre la implicación motivacional de los alumnos durante el desarrollo de la clase.

En este sentido, Solana (2005) señala que «la cesión de funciones docentes a los alumnos puede aportarnos grandes beneficios para incrementar la vinculación del alumno con el aprendizaje, evitando así situaciones de inhibición o falta de motivación» (pp.79).

También es interesante observar que Cervelló, Del Villar, Jiménez, Ramos, \& Blázquez (2003), observarón que los criterios de éxito que adoptan los discentes en las clases de educación física se relacionan de forma clara con la percepción de los climas motivacionales.

El presente trabajo tiene como objetivo genérico observar la situación de la Educación Física (EF) como disciplina mediante una indagación basada en la experiencia y los conocimientos de los profesionales en activo.

Para ello se establecieron una serie de objetivos específicos:

Indagar qué estrategias y procesos formativos se deberían de seguir en la EF para obtener un mejor rendimiento en el alumnado y una mejor capacitación del profesorado.

- Conocer cómo se puede obtener una mejor implicación del alumnado.

Saber qué perfil debería tener el profesorado EF en la actualidad.

\section{Material y Método}

Lametodología utilizada es de carácter cualitativo(Denzin \& Lincoln, 2000; Íñiguez, 1995) dentro del paradigma interpretativo (Goetz \& Le Compte, 1985; Albert, 2007), donde el reto es dar sentido a una cantidad de datos, con la intención de reducir el volumen de información, identificando pautas significativas y construir un marco para comunicar la esencia de lo que revelan los hechos en cuestión (Patton 2002), para comprender e interpretar la realidad del estudio investigado (Quintanal \& García, 2012).

\section{Muestra}

Responde al objetivo de nuestra investigación, habiendo sido el procedimiento de selección y acceso de conveniencia, con objeto de obtener la mejor información en el menor tiempo posible, de acuerdo a las circunstancias concretas que rodean tanto a los investigadores como a los sujetos encuestados (accesibilidad, rapidez, coste). Martínez-Salgado (2012). Para su selección se opta por los centros docentes que colaboran con la Facultad de Educación y Psicología de la UdG concretamente en el $4^{\circ}$ curso del Grado de maestro/a de Primaria, mención de EF.

Desde una perspectiva cualitativa, no se aspiraba a la representatividad de la muestra, pero sí a una mayor transferibilidad de los resultados, de modo que la información obtenida pudiera ser de más utilidad para otras poblaciones y contextos al proceder de una muestra suficientemente amplia y variada. Asimismo, por criterios éticos de la investigación se ha preservado el anonimato de los entrevistados y ocultado los datos concretos de sus centros de trabajo.

Para realizar este estudio se explicó a la dirección de cada uno de los centros en qué consistía la investigación y se solicitó por escrito a la institución y al profesorado de EF el permiso pertinente así como el uso de sus resultados. Consecuentemente, los datos se muestran siguiendo los principios éticos de selección y anonimato.

El proceso de recogida de datos constó de tres fases, la primera a través de un contacto telefónico directo con los profesores de EF con objeto de localizar los correos electrónicos de los mismos, dato imprescindible para hacerles llegar el cuestionario. La segunda, consistió en enviarles por correo electrónico la presentación de la investigación y el acceso al cuestionario. En la tercera fase se envió un segundo correo electrónico de recordatorio a fin de poder recoger el mayor número de datos posibles.

La muestra que finalmente contestó el cuestionario fue de 36 profesores de EF de los que 17 son hombres (47.2\%) y 19 mujeres (52.8\%). Respecto al tipo de centro al que pertenecían los profesores de la muestra encontramos que 31 (86.1\%) trabajaban en un centro público mientras que los 5 restantes lo hacen en centros concertados (13.9\%). Consecuentemente la mayoría (22) son funcionarios (61.1\%), 10 son interinos (27.8\%) y 4 son contratados (11.1\%).

La mayoría de los centros (29) se encuentran ubicados en la provincia de Girona y los 7 restantes en la de Barcelona. En la tabla 1 se puede observar la distribución por comarcas. La mayoría son del Gironés, Ampordán y de la Selva.

\begin{tabular}{|c|c|c|}
\hline & Frecuencia & Porcentaje \\
\hline Alt Empordà & 6 & 16.7 \\
\hline Baix Empordà & 4 & 11.1 \\
\hline Berguedà & 1 & 2.8 \\
\hline El Barcelonès & 1 & 2.8 \\
\hline El Maresme & 2 & 5.6 \\
\hline El Ripollès & 1 & 2.8 \\
\hline Gironès & 12 & 33.4 \\
\hline La Selva & 6 & 16.7 \\
\hline Vallès Oriental & 3 & 8.3 \\
\hline \multicolumn{3}{|l|}{$\begin{array}{l}\text { Tabla } 2 . \\
\text { Años de experiencia. }\end{array}$} \\
\hline & Frecuencia & Porcentaje \\
\hline Menos de 2 años & 1 & 2.8 \\
\hline Entre 3 y 8 años & 14 & 38.9 \\
\hline Entre 9 y 14 años & 12 & 33.3 \\
\hline Entre 15 y 20 años & 5 & 13.9 \\
\hline Más de 20 años & 4 & 11.1 \\
\hline
\end{tabular}

En relación al nivel de estudios impartidos, 33 docentes impartían primaria (91.7\%) y los 3 restantes (8.3\%) lo hacían en la ESO.

Finalmente, como se puede observar en la tabla 2, la mayoría de los docentes tienen entre 3 y 14 años de experiencia (72.2 si bien la franja comprendida entre 3 y 8 años es la de mayor frecuencia (38.9\%).

\section{Instrumento}

Se ha aplicado un cuestionario ad hoc a 36 profesores de EF con la finalidad que evidencien los problemas reales de enseñanza, así como la importancia y el impacto social que tiene la EF como materia educativa.

La elaboración del cuestionario de preguntas abiertas se ha diseñado partiendo de las cuestiones planteadas por Romero (2007) que buscaban indicios sobre la delimitación del campo didáctico de la EF y su actividad científica. En este cuestionario se recogen las preguntas que el autor se plantea para docentes de la EF en activo y se analizan con el objeto de tener una amplia visión del estado actual de la EF desde la perspectiva del profesorado. El cuestionario está formado por 7 preguntas abiertas (Tabla 3)

El objetivo de la aplicación de la encuesta responde al objeto de este estudio, obtener datos en relación a los problemas reales de enseñanza y aprendizaje de la EF, así como las preocupaciones y las prácticas reales de los docentes, y conocer la importancia y el impacto social que 
Tabla 3.

stinario abierto

¿Cuáles serían las grandes innovaciones conceptuales en EF que podrían ayudar a resolver los problemas y anomalías detectadas en el ámbito educativo?

¿Qué características presenta la práctica del profesorado para que los alumnos y alumnas tengan éxito en las clases de EF?

¿Qué condiciones se dan en la clase de EF para que el alumnado se implique, adquier compromiso de participación y le de sentido a las prácticas corporales?

¿Cuáles son las condiciones y los factores de enseñanza y aprendizaje que promueven el éxito de la EF como materia curricular?

¿Cómo sería la formación inicial que propiciaría la capacitación y cualificación ideal para el desempeño profesional?

¿Qué estrategias y procesos formativos habría que seguir para que el profesorado pudiera adecuarse a los cambios y a los retos que van surgiendo, de manera que llevaran a cabo unas buenas prácticas docentes?

¿Cómo sería la formación inicial que propiciaría la capacitación y calificación ideal para el ejercicio profesional de un maestro de EF?

puede tener la EF como materia educativa. Finalmente se procedió a dar un feedback sobre los resultados a los participantes.

\section{Análisis de Resultados}

Para realizar el análisis de los datos se han utilizado instrumentos cualitativos y cuantitativos. Primeramente, se han categorizado a través del programa Atlas.ti 7 para Windows (software para el análisis cualitativo de Datos) y posteriormente se ha completado con el programa SPSS versión 21 que otorga un aspecto más cuantitativo a la investigación. El análisis de tipo cualitativo ha permitido capturar, transcribir y ordenar información, así como codificar e integrar significado (Miles \& Huberman 1994).

Cabe destacar que el cuestionario de preguntas abiertas permitía dar más de una respuesta a cada pregunta y esto queda reflejado en los resultados obtenidos. La utilización de este cuestionario permitió describir, interpretar y contrastar diferentes valoraciones, no extrayendo conclusiones generalizables sino aproximación a unas realidades y necesidades concretas en relación al objeto de estudio.

La valoración de los resultados se presenta siguiendo el orden de las preguntas del cuestionario abierto.

\section{Resultados}

¿Cuáles serían las grandes innovaciones conceptuales en $\mathbf{E F}$ que podrían ayudar a resolver los problemas y anomalías detectadas en el ámbito educativo?

A continuación, se señalan los aspectos más destacados por los encuestados. La mayoría indica la importancia del trabajo en valores y cooperativo, así como el trabajo en competencias básicas (tabla 4):

«Trabajo de valores: cooperación, respeto, empatía... a través del juego» (caso 21); «la cooperación, respeto, solidaridad y uno de los más importantes, la autoestima» (caso 6); «el aprendizaje entre iguales» (caso 25); «la implantación de las competencias básicas en la educación aporta una visión más útil para la vida y una formación más contextualizada» (caso 35).

Como ventajas citan que:

El trabajo en valores «a partir de la práctica deportiva permite un desarrollo integral de nuestros alumnos» (caso 4).

«Através de un trabajo cooperativo, como podría ser el aprendizaje entre iguales, se podría favorecer la inclusión de los alumnos con más necesidades, además de su socialización, interacción e integración al grupo» (caso 25).

- Los alumnos gracias al trabajo cooperativo «asumen diferentes roles y aprenden a respetar los roles de los otros compañeros/as» (caso 7).

La implantación de las competencias básicas a la educación ya que según ellos «cambia la perspectiva de esta, dando más importancia a lo que viven los alumnos que a lo memorizan» y que también aportan «una visión más útil para la vida y una formación más contextualizada» (caso 27).

Por lo que hace referencia al tamaño de los grupos cooperativos se opta por grupos pequeños ya que «para llegar a un objetivo común es una buena herramienta para establecer interacciones respetando las diferencias y desarrollar habilidades sociales; Fomentando la discusión en grupo, el diálogo, la generosidad, el conflicto cognitivo, etc.
Permite a los alumnos aprender diferentes puntos de vista que le permiten rectificar y consolidar aprendizajes alcanzados» (caso 2).

¿Qué características debe presentar la práctica del profesorado para que los alumnos tengan éxito en las clases de EF?

La respuesta más repetida (tabla 5) es que las prácticas han de ser motivadoras y reflexivas. Se trata de hacer prácticas atractivas para los alumnos «para que se motiven al mismo tiempo que aprenden valores del juego, así como los diferentes aprendizajes de la asignatura» (caso 20). Esta motivación «se puede obtener, por ejemplo, a través de practicar deportes que les sean conocidos o cercanos, realizar ejercicios que posteriormente puedan exhibir ante otros grupos de la escuela...» (caso 20). Para ello se aconseja «presentar unidades didácticas motivadoras, valorando el esfuerzo individual y colectivo» (caso 23) y «cooperativas, expresivas, de acondicionamiento físico, al aire libre...» (caso 16). Velando por un buen desarrollo de capacidades coherentes con los objetivos planteados.

\begin{tabular}{lcc}
$\begin{array}{l}\text { Tabla } 4 . \\
\text { Innovaciones conceptuales EF. }\end{array}$ & & \\
\hline Trabajo en valores y actitudes & 12 & $24.49 \%$ \\
Nuevas estrategias de aprendizaje & 10 & $20.41 \%$ \\
Trabajo en las competencias básicas de la EF y desarrollo competencial & 8 & $16.33 \%$ \\
para la vida & 7 & $14.29 \%$ \\
Trabajo cooperativo & 3 & $6.12 \%$ \\
Trabajo en emociones & 3 & $6.12 \%$ \\
Trabajo en los hábitos saludables & 3 & $6.12 \%$ \\
Transversalidad de los aprendizajes & 2 & $4.08 \%$ \\
Colaboración con los tutores de otras especialidades & 1 & $2.04 \%$ \\
Aprendizaje significativo & & \\
& & \\
Tabla 5 & & \\
Características que presenta la práctica para tener éxito. & 9 & $17.65 \%$ \\
Prácticas motivadoras y reflexivas & 8 & $15.69 \%$ \\
Proporcionar experiencias motrices variadas y contextualizadas & 7 & $13.73 \%$ \\
Conocer los puntos fuertes y débil del grupo clase & 7 & $13.73 \%$ \\
Utilizar metodologías activas & 4 & $7.84 \%$ \\
Crear una atmosfera de trabajo en el aula & 4 & $7.84 \%$ \\
Adaptación a las características de los alumnos & 4 & $7.84 \%$ \\
El profesor como guía del proceso de enseñanza y buen comunicador & 3 & $5.88 \%$ \\
Trabajo cooperativo & 2 & $3.92 \%$ \\
Favorecer la autoestima & 1 & $1.96 \%$ \\
Profesores reflexivo & 1 & $1.96 \%$ \\
Profesor investigador de su propia práctica docente & 1 & $1.96 \%$ \\
Formación continuada de los docentes & &
\end{tabular}

¿Quéestrategias y procesos formativos habría que seguir paraque el profesorado pudiera adecuarse a los cambios y los retos que van surgiendo, de modo que llevaran a cabo unas buenas prácticas docentes?

Como se puede observar en la tabla 6, encontramos que el porcentaje más elevado considera aprendizaje continuo ya que para estar al día «es básico para ofrecer una buena calidad de enseñanza que todos los maestros se formen y se reciclen, ya que esto lleva a que se den clases mucho más precisas y mucho más reales a las necesidades de los alumnos y de la sociedad» (caso 5). Pero también se hace hincapié en que esta formación se adecuada «los procesos formativos de los docentes, que en muchas ocasiones no se varía o no avanza de la misma manera que lo hacen nuestros alumnos» (caso 7). En relación al tipo de formación no se opta por una en concreto: masters, cursos, talleres, seminarios... El tipo de formación también puede ir ligado al tipo de alumno. En este sentido González y Macías (2018) indican que los docentes valoran positivamente la formación permanente como herramienta para mejorar la intervención del maestro de educación física con alumnado con discapacidad.

En segundo lugar, también encuentran muy importante el intercambio de experiencias entre maestros de EF «para mejorar la metodología y recursos en el aula» (caso 2). Este intercambio se puede realizar o bien de forma restringa dentro de la misma escuela «colaboración entre escuelas, intercambios de profesionales, trabajos conjuntos..., que faciliten la comunicación e intercambio de experiencias» (caso 18) o bien interactuando con otros centres. También tenemos estudios como el de Martínez, Romero y Delgado (2011) en que los docentes valoraban positivamente la colaboración entre el profesor de Educación Física y los profesores de otras asignaturas para mejorar la salud del adolescente.

Se ha aplicado la prueba de Chi cuadrado entre algunas características que debería tener la práctica docente para tener éxito y algunas 
Tabla 6

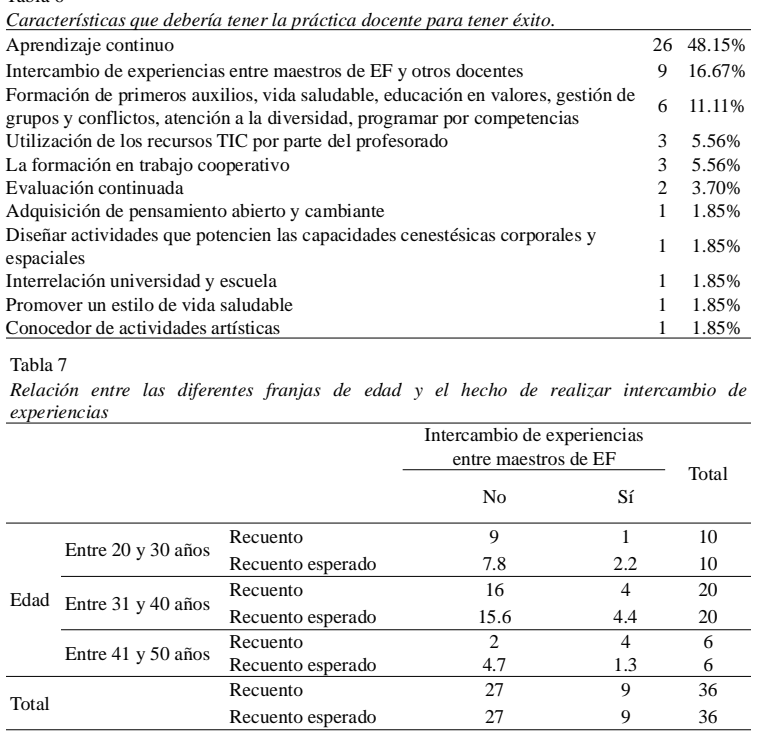

variables demográficas. Se han encontrado diferencias significativas entre la variable intercambio de experiencias entre maestros de EF y las diferentes franjas de edad ( $\mathrm{P}=.029$ ), siendo los mayores los que están más a favor de realizar intercambios de experiencias (Tabla 7).

También se han generado diferencias entre las variables comarca y la realización del aprendizaje continuo ( $\mathrm{p}=.026)$. Los que están más a favor del aprendizaje continuo son los profesores de EF del Gironés, Alt Empordá, Maresme, La Selva, El Bergadá y el Barcelonés.

Tabla 8

Relación entre las comarcas y el hecho estar a favor del aprendizaje continuo

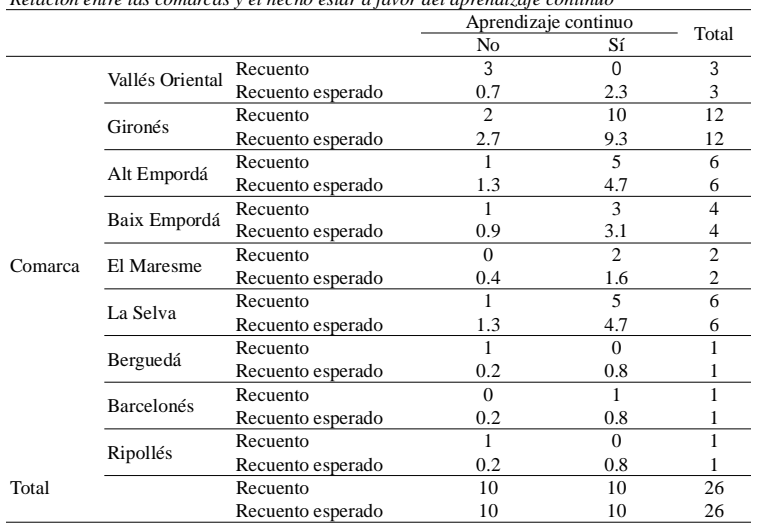

¿Qué condiciones se dan en la clase de EF para que todos los alumnos se impliquen, adquieran compromiso de participación y les den sentido a las prácticas corporales?

Por una parte, destaca el hecho de que la mayoría de las respuestas se decantan por un aprendizaje significativo.

«El aprendizaje significativo es la base de este compromiso. Buscar el temario adecuado que motive a los alumnos y les haga trabajar de la mejor manera posible adecuando la enseñanza al nivel e interés de los alumnos» (caso 1$)$.

En este sentido sería interesante llevar a cabo «actuaciones que pueden percibir directamente los resultados, de lo que están haciendo es útil y les servirá» (caso 2). Para ello consideran que «las actividades propuestas por parte del profesorado deben atender a la diversidad de los alumnos con una variedad de metodología y recursos que intente llegar a todo el alumnado para motivar y conseguir de ellos una actitud positiva hacia la asignatura» (caso 28). También indican que se debe aprovechar el hecho de que «los alumnos suelen ir a las clases de EF con mucha motivación, ya que esta es una asignatura que se diferencia mucho al resto de asignaturas y que les da mucha libertad, ya sea de movimiento y o de imaginación» (caso 5).

Por otra parte, como segunda opción escogida, encontramos el trabajo cooperativo.

«Cuando te sientes parte de un grupo/equipo sabes de la responsabilidad propia que tienes en la incidencia de éste. De esta manera, con la cooperación y el trabajo en equipo es más fácil implicar a todo el mundo.» (caso 26). Además, algunos docentes señalan que «El trabajo en pequeños y grandes grupos, heterogéneos, cambiantes, mixtas» (caso 21).

Finalmente, también se recomienda la participación por parte de todos los alumnos:

«Que todos los alumnos sean participativos y cada uno pueda aportar aspectos positivos en función de las habilidades individuales de cada uno. La suma de todo el alumnado es el que tiene que dar la riqueza de las prácticas corporales» (caso 7).

Dicha participación se puede fomentar a través del trabajo en grupo «los juegos cooperativos son la mejor herramienta para que todos los alumnos se impliquen en la actividad y se sientan incluidos» (caso 10).

El resto de opciones que destacan son la participación de todos los alumnos y el buen fomento de la práctica física y deportiva.

\begin{tabular}{lcc} 
Tabla 9. & \\
Condiciones y los factores de enseñanza y aprendizaje que promueven el éxito & de EF. \\
\hline Aprendizaje significativo (motivación) & 20 & $34.48 \%$ \\
Trabajo cooperativo & 14 & $24.14 \%$ \\
Fomentar la participación de todos los alumnos & 9 & $15.52 \%$ \\
un buen fomento de la Práctica física y deportiva motriz variada & 7 & $12.07 \%$ \\
Aprendizaje a través del juego & 2 & $3.45 \%$ \\
Clima de trabajo relajado & 2 & $3.45 \%$ \\
Promover metodologías activas & 2 & $3.45 \%$ \\
Tener en cuenta los diferentes niveles de los alumnos & 2 & $3.45 \%$ \\
\hline & & \\
Tabla 10. & \\
Condiciones y factores de enseñanza y aprendizaje que promueven el éxito de & EF. \\
\hline La motivación y creatividad & 7 & $15.56 \%$ \\
Trabajar en grupo & 6 & $13.33 \%$ \\
Contenidos atractivos y adaptados a las necesidades de los alumnos & 5 & $11.11 \%$ \\
Conocer y desarrollar las propias capacidades & 4 & $8.89 \%$ \\
El juego como medio de desarrollo de las capacidades cognitivas, & 4 & $8.89 \%$ \\
creativas, motoras, afectivas y de socialización & 4 & 3 \\
Reconocimiento e implicación de la comunidad educativa & $3.67 \%$ \\
Atención a la diversidad & 2 & $4.44 \%$ \\
La autoestima & 2 & $4.44 \%$ \\
Crear un buen clima de aprendizaje & 2 & $4.44 \%$ \\
Organización de materiales, recursos y espacios & 2 & $4.44 \%$ \\
La utilización del movimiento como fuente de placer y de aprendizaje & 2 & $4.44 \%$ \\
Utilización de diferentes metodologías activas y estilos de aprendizaje & 2 & $4.44 \%$ \\
Aprender mediante la experimentación & 2 & $4.44 \%$ \\
Diversificar los procedimientos de evaluación & 1 & $2.22 \%$ \\
Interdisciplinariedad & 1 & $2.22 \%$ \\
\hline
\end{tabular}

¿Cuáles son las condiciones y los factores de enseñanza y aprendizaje que promueven el éxito de la EF como materia curricular?

De las respuestas obtenidas y clasificadas (Tabla 10) se observa que la mayoría optan por la motivación como factor y condición que promueve el éxito de la EF como materia curricular.

Si el profesorado es la clave en la mejora cualitativa de los sistemas educativos, deberíamos determinar ¿Cómo debe ser el perfil del maestro que necesita la escuela hoy?

Dentro del perfil que debería tener el maestro de EF el más valorado es saber trabajar en equipo, seguido de tener empatía. Esto supone diferentes ventajas como «Colaborar con otros profesionales (otros maestros de la escuela, EAP...) permite atender las necesidades individuales de cada niño» (caso 22) y permitir «al centro ir en una misma línea pedagógica» (cas 23).

También se valora el hecho de que utilice proyectos de innovación educativa. «El maestro debe ser dinámico, estar al día de las nuevas prácticas en educación física» (caso 10). Esto conlleva que disponga de «una mentalidad abierta que sea capaz de adaptarse a los cambios» (caso 5) y unas «ganas de aprender y adaptarse continuamente a las nuevas necesidades educativas, que cambian a marchas forzadas (nuevas tecnologías, recortes...)» (caso 25). Pero esta innovación se debería de aplicar «siempre y cuando ello beneficie su tarea docente y el aprendizaje de sus alumnos» (caso 23).

Finalmente, también se valora el hecho de que sea flexible y se adapte a las condiciones. Se tiene que saber adaptar «a las necesidades ya sea de la escuela o en las necesidades de los alumnos, que se producen 
en cada momento» (caso 5). Si se «adapta a la realidad de alumnado que tiene y adapta las prácticas corporales a este alumnado para conseguir el máximo de cada uno» (caso 7).

Tabla 11

Perfil del maestro que necesita la escuela hoy.

\begin{tabular}{lcc}
\hline Saber trabajar en equipo & 11 & $16.92 \%$ \\
Tener empatía & 6 & $9.23 \%$ \\
Trabajar con proyectos de innovación educativa & 5 & $7.69 \%$ \\
Tener habilidades para proporcionar las herramientas necesarias & 5 & $7.69 \%$ \\
Ser persona activa e interesada en su trabajo & 5 & $7.69 \%$ \\
Potenciar actitudes y valores & 4 & $6.15 \%$ \\
Tener una base sólida de conocimientos & 4 & $6.15 \%$ \\
Atender a la diversidad & 3 & $4.62 \%$ \\
Formación constante para adaptarse a los cambios & 3 & $4.62 \%$ \\
Saber escuchar & 3 & $4.62 \%$ \\
Ser observador & 3 & $4.62 \%$ \\
Tener capacidad de reflexión & 3 & $4.62 \%$ \\
Tener una actitud crítica & 2 & $3.08 \%$ \\
Ser investigador & 2 & $3.08 \%$ \\
Saber adaptarse a los alumnos y a los cambios & 2 & $3.08 \%$ \\
Tener capacidad de análisis & 1 & $1.54 \%$ \\
Hacer de intermediario entre contenidos y alumnos & 1 & $1.54 \%$ \\
Ser motivador & 1 & $1.54 \%$ \\
Un líder & 1 & $1.54 \%$ \\
\hline
\end{tabular}

¿Cómo sería aquella formación inicial que propiciaría la capacitación y calificación ideal para el ejercicio profesional de un maestrode EF?

La mayoría de las respuestas se decantan por el hecho de potenciar les prácticas en las escuelas para adquirir experiencia.

«Como en la gran mayoría de trabajos, la experiencia es fundamental. Para que un maestro esté capacitado para afrontar ante el grupo clase, lo que necesita son horas de vuelo» (caso 7)

Además, resaltan su importancia debido a que «el bagaje práctico de aquel maestro llevado a cabo en la escuela y en activo (prácticas in situ). Y de la práctica es de donde aparecen situaciones a partir de las cuales podemos indagar y profundizar en los aspectos relevantes que caracterizan al maestro de Educación Física; adaptación a diferentes realidades, diferente alumnado, diferente metodología, diferente tipología de escuela, etc.» (caso 18). También se remarca que dichas prácticas tienen que realizarse «bajo la supervisión de un maestro experimentado que controle dé apoyo al proceso» (caso 8).

La segunda opción es la de que las asignaturas que se impartan sea de carácter práctico, es decir, «una formación que tenga una estrecha vinculación en la práctica docente futura. Aunque no puede olvidarse una base teórica firme pero que sea significativa y que se pueda vincular a la práctica para que no quede como una teoría lejana a la realidad» (caso 3). Esto permitirá tener «una formación más real, más adaptada al día a día de las escuelas y en las necesidades y dificultades de los alumnos, con más casos reales, más tiempo de práctica en las escuelas y sobre todo más formación con un maestro que esté trabajando día a día en una escuela» (caso 6).

Finalmente, también cabe resaltar la valoración de un buen conocimiento de la anatomía ya que esto permite «la adquisición de aquellos conocimientos habilidades y competencias relacionadas con el cuerpo y su actividad motriz que contribuyen al desarrollo integral de la persona y la mejora de la calidad de vida» (caso 35)

\begin{tabular}{|c|c|c|c|c|c|}
\hline \multicolumn{5}{|l|}{ Tabla 12} & Formación inicial que propiciaría la capacitación y calificación ideal. \\
\hline \multicolumn{4}{|c|}{ Potenciar la practica en la escuela } & 16 & $53.34 \%$ \\
\hline \multicolumn{4}{|c|}{ Desarrollar la competencia motriz } & 6 & $20.00 \%$ \\
\hline \multicolumn{4}{|c|}{ Un buen conocimiento de la anatomía } & 3 & $10.00 \%$ \\
\hline \multicolumn{4}{|c|}{ Formación permanente del docente } & 3 & $10.00 \%$ \\
\hline \multicolumn{4}{|c|}{ Establecer relaciones bilaterales entre universidad y escuela } & 1 & $3.33 \%$ \\
\hline \multicolumn{4}{|c|}{ Debería dar un base sólida sobre teorías de educación } & 1 & $3.33 \%$ \\
\hline \multicolumn{6}{|c|}{$\begin{array}{l}\text { Tabla } 13 \\
\text { El desarrollo de la competencia motriz en función del tipo de centro }\end{array}$} \\
\hline \multicolumn{6}{|c|}{$\begin{array}{l}\text { El desarrollo de la competencia motriz en función del tipo de centro } \\
\qquad \begin{array}{c}\text { Desarrollo de la competencia } \\
\text { motriz }\end{array}\end{array}$} \\
\hline & & & No & Sí & \\
\hline \multirow{4}{*}{ Tipo Contrato - } & \multirow[b]{2}{*}{ Centro Público } & Recuento & 28 & 3 & 31 \\
\hline & & $\begin{array}{l}\text { Recuento } \\
\text { Esperado }\end{array}$ & 25.8 & 5.2 & 31 \\
\hline & \multirow[b]{2}{*}{ Centro concertado } & Recuento & 2 & 3 & 5 \\
\hline & & $\begin{array}{l}\text { Recuento } \\
\text { Esperado }\end{array}$ & 4.2 & 0.8 & 5 \\
\hline \multirow{2}{*}{\multicolumn{2}{|c|}{ Total }} & Recuento & 30 & 6 & 36 \\
\hline & & $\begin{array}{l}\text { Recuento } \\
\text { Esperado }\end{array}$ & 30 & 6 & 36 \\
\hline
\end{tabular}

Se ha aplicado la prueba de chi cuadrado entre tipo de centro y desarrollo de la competencia motriz (tabla 13) y se observan diferencias significativas, siendo en los centros concertados donde existe un mayor desarrollo de la competencia motriz $(\mathrm{p}=.031)$.

\section{Discusión y Conclusiones}

En este artículo se analiza el conocimiento didáctico y la práctica profesional desde la perspectiva de un grupo de profesores de EF en activo de centros docentes que colaboran con la Facultad de Educación y Psicología de la, UdG, concretamente en el prácticum de Grado de maestro/a de Primaria mención EF. En este apartado se discute la información obtenida y se identifican los aspectos más destacables.

En relación al objetivo de investigación marcado se ha concluido que los docentes se centran en la motivación como el factor imprescindible que promueve el éxito de la EF escolar. Por otra parte, abogan por unas prácticas motrices motivadoras, reflexivas y cooperativas. Las prácticas deben ser contextualizadas y responder a los intereses de los alumnos, favoreciendo la creación de un buen clima en el aula. Del análisis de los resultados se extrae que los docentes consideran que, para poder llevar a cabo una docencia de calidad, la EF debe ser innovadora, motivadora y crítica, que potencie la motricidad aplicando metodologías activas, dinámicas y reflexivas desde un enfoque transversal con el fin de potenciar y desarrollar competencias, capacidades y habilidades para la vida. Centrando la innovación de la EF en la importancia del trabajo en valores, y cooperativo, así como el trabajo en competencias básicas, en lo que coincide con los aspectos destacados en estudios como los de Contreras \& Cuevas, 2011; Zamorano, 2011; Cañabate \& Zagalaz 2010, entre otros.

La formación continua es un elemento destacado por los docentes con el objeto de ofrecer una buena calidad de enseñanza, generando diferencias entre los encuestados de las distintas comarcas, donde los del Gironés son los que están más a favor. También se observa que los maestros de EF de mayor edad son los que aprecian más el intercambio de experiencias como un aspecto primordial en el proceso continúo con objeto de poder llevar acabo buenas prácticas docentes.

En relación a las condiciones y a los factores de enseñanza y aprendizaje que promueven el éxito de EF, los docentes resaltan la motivación, el trabajo cooperativo, la inclusión de todos los alumnos a través de prácticas motrices variadas, el juego como medio de aprendizaje, utilizando metodologías actives donde el alumno es el protagonista de su aprendizaje. Resultados que también són compartidos con otros estudios como los llevado a cabo por (Méndez- Alonso, Fernández-Río, Méndez-Giménez, \& Prieto ,2015; Baena-Extremera \& Granero-Gallegos, 2012 y Romero, 2007)

A nivel curricular destacan los docentes que comentan que para tener éxito en su desarrollo es necesario promover contenidos atractivos y adaptados a las necesidades de los alumnos a través del trabajo en grupo, que facilite el desarrollo de competencias y capacidades cognitivas, creativas, motoras, afectivas y de socialización.

Los resultados como se puede apreciar son muy compartidos con la mayoría de los encuestados, encontrando diferencias significativas entre la variable intercambio de experiencias entre maestros de $\mathrm{EF}$ y las distintas franjas de edad ( $\mathrm{P}=.029)$, siendo los mayores los que están más a favor de realizar intercambios de experiencias. Así como también se han generado diferencias entre la realización del aprendizaje continuo $(\mathrm{P}=.026)$. Los que están más a favor son los del Gironés, Alt Empordá, Maresme, La Selva, El Bergadá y el Barcelonés.

El estudio se presenta tiene como innovación conocer e indagar el conocimiento didáctico los propios docentes en activo con objeto de conocer cuáles son los nuevos retos y estrategias a tener en cuenta para poder llevar a cabo una docencia de calidad de la Educación física en el siglo XXI con docenes competentes que puedan dar respuestas a las necesidades de los actuales estudiantes. Para ello es necesario transformar la escuela para saber más, para ser más competente, para ser más persona.

Es imprescindible llevar a cabo una formación competencial conti- 
nuada de los docentes a través de cursos, seminarios... sobre el trabajo cooperativo, en una educación en valores, de gestión de grupos, atención a la diversidad... Una formación de calidad que permita llevar a cabo procesos críticos, reflexivos y constructivos hacia la mejora docente, principalmente en el saber hacer de maestro de educación Física. Para ello creemos que uno de los elementos importantes a tener en cuenta, es el llevar a cabo un trabajo de estrecha colaboración entre estudiantes, profesorado de la Facultad de la mención de Educaicón Física y maestro de EF en activo con el fin de compartir proyectos de innovación docente. Otro aspecto a tener en cuenta son los encuentros de profesionales donde se puede intercambiar experiencias entre maestros de EF y otros docentes. Es decir crear espacios de intercambio y de análisis de experiencias y propuestas que aporten buen conocimiento didáctico y una práctica profesional de calidad. Donde la profesionalidad del docente pueda revertir en un ecosistema educativo inclusivo y de calidad que permita desarrollar alumno-persona asertivo, competente y divergente (Colomer, 2017).

\section{Referencias}

Albert, M.J. (2007). La investigación educativa. Barcelona: McGrawHill.

Aubert, A., Bizkarra, M. \& Calvo, J. (2014). Actuaciones educativas de éxito desde la Educación Física. Retos. Nuevas tendencias en EF, Deporte y Recreación, 25, 144-148. .

Baena-Extremera, A. \& Granero-Gallegos, A. (2012). Competencias profesionales en Educación Física y necesidades educativas. Espiral. Cuadernos del Profesorado, 5(10), 105-109.

Balázs, F., Susan, C. Dancs, H, \& József, B. (2016). Satisfaction and preferences of PE students and the head of the PE department: Meeting the new curricular expectations. Journal of Human Sport and Exercise 11(1), 1-18.

Blasco, J., Romero, C., Mengual, S., Andrés B., Fernández Revelles, A.B., Delgado, M.A. \& Vega, L. (2011). Estilo de aprendizaje de los estudiantes de magisterio de educación física y ciencias del deporte de las universidades de Granada y Alicante. Cultura y Educación: Revista de teoría, investigación y práctica, 23(3), 371383.

Blázquez, D. \& Sebastiani, E. (2009). Enseñar por competencias en educación física. Barcelona: INDE Publicaciones.

Bisquerra, R. (2011). Educación física, competencias básicas y educación. EmásF: Revista digital de EF, 11, 4-6

Calderón, A., Martínez, D. \& Méndez, A. (2013). Formación permanente y percepción del profesorado sobre el desarrollo de las competencias básicas con el modelo de Educación Deportiva. Retos. Nuevas tendencias en EF, Deporte y Recreación, 23, 33-38.

Cañabate, D. \& Zagalaz, M.L. (2011). Aportaciones de la educación física al desarrollo de la competencia básica: autonomía e iniciativa personal. Tándem. Didáctica de la EF, 37, 69-77.

Cañabate, D. \& Zagalaz, M.L. (2010). Aportaciones del área de educación física a la competencia básica «Aprender a Aprender». Retos. Nuevas tendencias en EF, Deporte y Recreación, 18, 52-55.

Cañabate, D. (2010). Proposta de seqüenciació didàctica en relació a les aportacions del'àrea d'educació física a la competència comunicativa lingüística i audiovisual. Comunicació Educativa, 23, 21-28.

Cervelló, E., Del Villar, F., Jiménez, R., Ramos, L., \& Blázquez, F. (2003). Clima motivacional en el aula, criterios de éxito de los discentes y percepción de igualdad de trato en función del género en las clases de Educación Física. Enseñanza, 21, 379-395.

Cervelló, E. M., Iglesias, D., Moreno, P., Jiménez, R. \& Del Villar, F. (2004). Aplicación de modelos de ecuaciones estructurales al estudio de la motivación de los alumnos en las clases de educación física. Revista de Educación, 335, 371-382.

Collazo, M. (2014). El cambio curricular, una oportunidad para repensar(nos). InterCambios, 1(1), 36-43.

Colomer, J. (2017). Danza en el aula: nuevos estudiantes, nuevas estrategias, nuevos lenguajes En D.Cañabate y A.Soler (coord.) Movi- miento y lenguajes. De la experiencia sensoperceptiva a la concienciayel pensamiento. Barcelona: Graò.

Contreras, O. \& Cuevas, R. (Coord) (2011). Las competencias básicas desde la educación física (Vol. 1). Barcelona: INDE.

Contreras, O.; Cuevas, R.; Blázquez, D.; Lleixà, T.; Garcia, L.M.; Sebastini, E.M. \& Barrachina, J. (2012). Las competencias del profesor de EF. Barcelona: INDE.

Del Valle, S., De la Vega, R. \& Rodríguez, M. (2015). Percepción de las competencias profesionales del docente de educación física en primaria y secundaria. Revista Internacional de Medicina y Ciencias de la Actividad Física y el Deporte, 15(59), 507-526.

Delicado, M., Trujillo, J.L \& García, L.M. (2018). Valoración sobre la formación en la mención deEducación Física, porparte del alumnado de Grado en Educación Primaria. Retos, 34, 194-199.

Dellinger, A.B., Bobbett, J., Olivier, D.F. \& Ellett, Ch.D. (2008). Measuring teathers' self-efficacy beliefs: Development and use of the TEBS-Self. Teaching and Teacher Education, 24(3), 751-766.

Denzin, N.K. \& Lincoln, Y.S. (2000). The discipline and practice of qualitative research. En N.K. Denzin \& Y.S. Lincoln (Eds.), Handbook of Qualitative Research, (1-28). London: Sage Publications.

Díaz, M. (2009). Percepción de competencia del profesorado de Educación Física e inclusión. Revista Internacional de Medicina y Ciencias de la Actividad Física y el Deporte, 9(35), 322-348.

Díaz del Cueto, M. \& Aguado, R. (2012). Percepción de competencia del profesorado de educación física con experiencia sobre la tarea como recurso didáctico. Retos. Nuevas tendencias en EF, Deportey Recreación, 22, 16-18.

García, M.J. (2010). Aportaciones al desarrollo de competencias básicas desde el área de educación física. Wanceulen: EF Digital, 6, 515.

Goetz, J.P. \&. LeCompte, M.D. (1985). Etnografia y diseño cualitativo en investigación. Madrid: Morata.

González, M.T. (2010). El alumno ante la escuela y su propio aprendizaje: algunas líneas de investigación en torno al concepto de implicación. Revista Iberoamericana sobre Calidad, Eficacia y Cambio en Educación ,8(4), 10-31.

González, S., García, L.M., Contreras, O. \& Sánchez Mora, D. (2009). El concepto de iniciación deportiva en la actualidad. Retos. Nuevas tendencias en EF, Deporte y Recreación, 15, 14-20.

González, I. \& Macias, D. (2018). La formación permanente como herramienta para mejorar la intervención del maestro de educación física con alumnado con discapacidad. Retos, 33, 118-122.

Gutierrez, M. (2014). Relaciones entre el clima motivacional, las experiencias en educación física y lamotivación intrínseca de los alumnos. Retos. Nuevas tendencias en Educación Física, Deporte y Recreación, 26, 9-14

Hernández, J.L. \& Velazquez, R. (2010). La educación Física a estudio. El profesorado, el alumnado y los procesos de enseñanza. Barcelona: Grao

Hernández, A., Díez, L.J., Pacheco, J.J. \& Sosa, G. (2008). Contribución de la materia de EF en la adquisición de competencias básicas en la etapa secundaria obligatoria. Actas al IVCongreso Internacional y XXV Nacional de Educación física. Cordoba.España. .

Íniguez, L. (1995). Métodos cualitativos en Psicología Social: Presentación. Revista de Psicología Social Aplicada, 5(1/2), 5-26.

Ley Orgánica 8/2013, de 9 de diciembre, para la mejora de la calidad educativa. BOE, num. 295, de 10 de diciembre.

Lleixá, T., Capllonch, M., \& González, C. (2015) Competencias básicas y programación de Educación Física. Validación de un cuestionario diagnóstico. Retos. Nuevas tendencias en EF, deporte y recreación, 27, 52-57.

Lleixá, T. (2007). Educación física y competencias básicas. Contribución del área a la adquisición de las competencias básicas del currículo. Tándem. Didáctica de la EF, 23, 31-37

López, J. (2010). Contribución de la EF a la adquisición de las competencias básicas en Bachillerato. EmásF: Revista Digital de EF, 6, 
$36-46$

López Pastor, Pérez, Manrique \& Monjas (2016). Los retos de la Educación Física en el Siglo XXI. RETOS. Nuevas Tendencias en Educación Física, Deporte y Recreación, 29, 182-187.

López, V., Pérez, D., Manrique, J.C. \& Monjas, R. (2016). Los retos de la Educación Física en el Siglo XXI. Retos, 29, 182-187.

Martínez-Salgado, C. (2012). El muestreo en investigación cualitativa: principios básicos y algunas controversias. Ciênc. Saúde coletiva, 17(3), 613-619.

Martínez A.C., Romero, C \& Delgado, M. (2011). Profesorado y padres ante la promoción de la actividad física del centro escolar. Revista Internacional de Medicina y Ciencias de la Actividad Física y del Deporte, 11 (42), 310-327.

Méndez Alonso, D., Fernández Río, J., Méndez Giménez, A., \& Prieto. J.A. (2015). Análisis de los currículos autonómicos LOMCE de Educación Física en Educación Primaria. Retos. Nuevas Tendencias en EF, Deporte y Recreación, 28, 15-20.

Méndez-Giménez, A., López-Téllez, G., \& Sierra-Arizmendiarrieta, B. (2009) Competencias básicas: sobre la exclusión de la competencia motriz y las aportaciones desde la Educación Física. Retos. Nuevas Tendencias en EF, Deporte y Recreación, 16, 51-57.

Monzonís, N. \& Campllonch, M. (2015). Mejorar la competencia social y ciudadana: innovación desde Educación Física y tutoría. Retos de la Actividad Física y el Deporte, 28, 256-262

Marchesi, A. (2007): Sobre el bienestar de los docentes. Competencias, emociones y valores. Madrid: Alianza Editorial.

Miles, M. \& Huberman, A.M. (1994). Data management and analysis methods, en Denzin, N.K. \& Lincoln, Y.S. (Ed.): Handbook of qualitative research, (429-444). London: Sage Publication.

Ministerio de Educación, Cultura y Deporte (2014). Real Decreto 126/ 2014, de 28 de febrero, por el que se establece el currículo básico de la Educación Primaria. Madrid: BOE 52 de 1 de marzo.
Morales, V., Berrocal, M.A., Morquecho, R. \& Hernández, A. (2013). Evaluación de la calidad en elárea de educación física en un centro de enseñanza secundaria y bachillerato. Revista Iberoamericana de Psicología del Ejercicio y el Deporte, 8(2), 411-427.

Patton, M.Q. (2002). Qualitative research \& evaluation methods $\left(3^{a}\right.$ ed.). Thousand Oaks, CA: Sage.

Quintanal, J. \& García, B. (2012). Fundamentos básicos de metodologia de investigación educativa. Madrid: CCS.

Romero, C. (2007). Delimitación del campo didáctico de la Educación Física y de su actividad científica. Profesorado. Revista de Currículum y Formación de Profesorado, 11(2), 1-45.

Skaalvik, E.M. \& Skaalvik, S. (2007). Dimensions of teacher selfefficacy and relations with strain factors, perceived college teacher efficacy, and teacher burnout. Journal of Educational Psychology, 99(3), 611-625.

Solana, A. (2005). Análisis de la evolución del pensamiento de los alumnos de Bachillerato sobre la aceptación de responsabilidades en las clases de Educación Física. Apunts. Educación Física y Deportes, 79, 29-39.

Vaca, M.J. (2008). Contribución de la Educación Física escolar a las competencias básicas señaladas en la LOE para la Educación Primaria. Tándem. Didáctica de la EF, 26, 52-61.

Vicente, M. (2011). Escuela y educación física en el contexto de la enseñanza por competencias. Reflexiones genealógicas desde la pedagogía crítica. Cultura, Cienciay Deporte, 6(18), 161-170.

Zagalaz, M.L., Cachón, J. \& Lara, A.J. (2008). La educación física en primaria a partir de la ley orgánica de educación (LOE, 2006). Especial atención a la enseñanza por competencias. Formación Continuada. Jaén: LOGOSS.

Zamorano, D. (2011). ¿Contribuciones del área de educación física al desarrollo de las competencias básicas o interdisciplinariedad? EmásF: Revista Digital de EF, 8, 59-73.

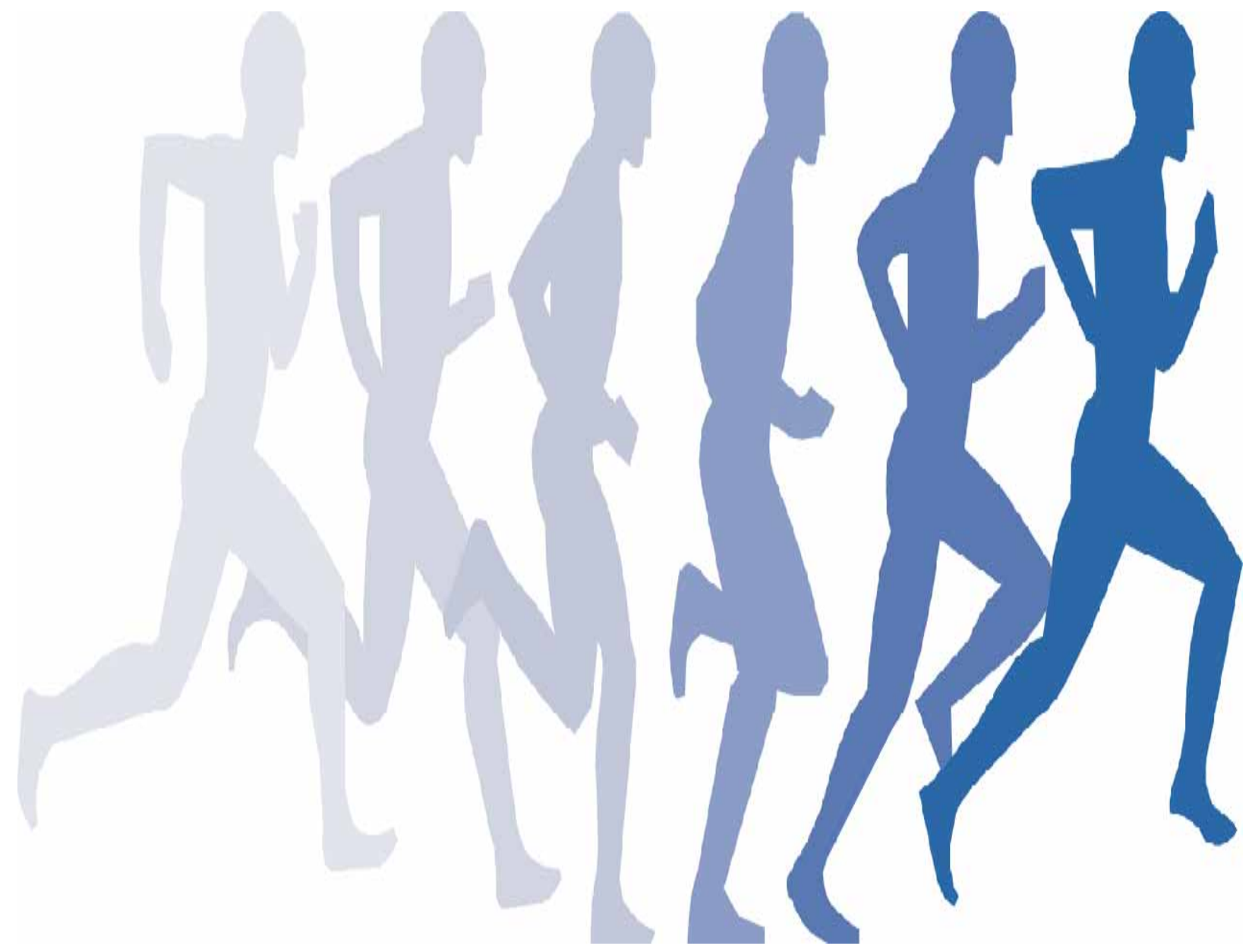

